\title{
FINDING OF INCOMES OF HM-NETWORK WITH LIMITED WAITING TIMES IN QUEUES BY MEANS OF THE SUCCESSIVE APPROXIMATIONS METHOD
}

\author{
Katsiaryna Kosarava ${ }^{1}$, Mikhail Matalytski ${ }^{2}$, Kiril Rozau ${ }^{1}$ \\ ${ }^{I}$ Grodno State University, Belarus \\ ${ }^{2}$ Institute of Mathematics, Czestochowa University of Technology, Poland \\ koluzaeva@gmail.com,m.matalytski@gmail.com
}

\begin{abstract}
The article is devoted to the successive approximations method combined with the series method and its application for finding of expected incomes in HM-network systems with limited waiting times in queues and one-type messages. The properties of successive approximations were researched. The example of implementation of the described method is calculated.
\end{abstract}

\section{Expected incomes of HM-network with limited waiting times in queues}

Let's consider the HM-network of arbitrary topology with one-type messages; the network consists of $n+1$ queueing systems (QS) $S_{0}, S_{1}, \ldots, S_{n}$ ( $S_{0}$ - external environment), in a general case the QS can be multiline and contains $m_{i}$ service lines, $i=\overline{1, n}$. The state of such network could be described by vector $k(t)=\left(k_{1}, k_{2}, \ldots, k_{n}, t\right)$, where $k_{i}$ - number of messages in system $S_{i}$ in the moment $t, i=\overline{1, n}$. The incoming flow arrives in the network with rate $\lambda$. Let's denote the service rate in system $S_{i}$ (in case when $k_{i}$ messages are in it) as $\mu_{i}\left(k_{i}\right) ; p_{0 j}$ probability of message transfer from the external environment to system $S_{j}$, $\sum_{j=1}^{n} p_{0 j}=1 ; p_{i j}$ - probability of message transfer to system $S_{j}$ after its service in system $S_{i}, \sum_{j=0}^{n} p_{i j}=1, i=\overline{1, n}$. When a message doesn't wait its service in system $S_{i}$ moves to QS $S_{j}$ with probability $q_{i j} ; \theta_{i}$ - rate of message leaving from queue of the $i$-th QS, $i=\overline{1, n}, j=\overline{0, n}$. Matrices $P=\left\|p_{i j}\right\|_{(n+1) \times(n+1)}$ and $Q=\left\|q_{i j}\right\|_{n \times(n+1)}$ are matrices of transition probabilities of irreducible Markov chains. Message which 
comes from one system to another brings to the last one some income, so the income of the first system descends on this value.

Let's determine $v_{i}(k, t)$ the total expected income which system $S_{i}$ receives during time $t$ if the network is in state $k$ in the initial moment. In the general case, when the network functioning is described by the Markov process $k(t), t \geq 0$, and incomes of message transitions between network states don't depend on time, system of difference-differential equations (DDE) for expected incomes of system $S_{i}$ can be written

$$
\frac{d v_{i}(k, t)}{d t}=\mathrm{A}_{i}(k)-\Lambda_{i}(k) v_{i}(k, t)+\sum_{c, s=1}^{n} \Phi_{i c s}(k) v_{i}\left(k+I_{c}-I_{s}, t\right),
$$

where: $\Lambda_{i}(k), \Phi_{i c s}(k)$ - some limited non-negative functions. The count of equations in system (1) equals the number of network states.

In the present article we observe the exponential HM-network with limited waiting times of messages in queues. Let $r_{i}(k)$ - income of the system $S_{i}$ in unit time if the network is in state $k ;-R_{i 0}\left(k-I_{i}, t\right),-H_{i 0}\left(k-I_{i}, t\right)$ - incomes of the system $S_{i}$ which correspond to a change of network state from $(k, t)$ to $\left(k-I_{i}, t+\Delta t\right)$ in cases when the message moves to the external environment after its service in system and when the message doesn't wait service in system and moves from the queue to the external environment; $r_{0 i}\left(k+I_{i}, t\right)$ - income of system $S_{i}$, when network changes its state from $(k, t)$ to $\left(k+I_{i}, t+\Delta t\right)$ because of message transition from the external environment to this system; $r_{i j}\left(k+I_{i}-I_{j}, t\right)$ - the income of the system $S_{i}$, when the network changes its state from $(k, t)$ to $\left(k+I_{i}-I_{j}, t+\Delta t\right)$ because of message transition to $S_{i}$ after its service in $S_{j}, h_{i j}\left(k+I_{i}-I_{j}, t\right)$ - the income of the system $S_{i}$, when the network changes its state from $(k, t)$ to $\left(k+I_{i}-I_{j}, t+\Delta t\right)$ when the message doesn't wait its service in system $S_{j}$ and moves from the queue to system $S_{i}$. Then the following statement is satisfied.

Theorem 1. System of DDE for expected incomes of system $S_{i}$ has the form

$$
\begin{aligned}
\frac{d v_{i}(k, t)}{d t}=-\left[\lambda+\sum_{j=1}^{n}\left(\mu_{j} \min \left(k_{j}, m_{j}\right) u\left(k_{j}\right)+\theta_{j}\left(k_{j}-m_{j}\right) u\left(k_{j}-m_{j}\right)\right)\right] v_{i}(k, t)+ \\
+\sum_{j=1}^{n}\left\{\lambda p_{0 j} v_{i}\left(k+I_{j}, t\right)+\left[\mu_{j} \min \left(k_{j}, m_{j}\right) u\left(k_{j}\right) p_{j 0}+\right.\right.
\end{aligned}
$$




$$
\begin{gathered}
\left.\left.+\theta_{j}\left(k_{j}-m_{j}\right) u\left(k_{j}-m_{j}\right) q_{j 0}\right] v_{i}\left(k-I_{i}, t\right)\right\}+ \\
+\sum_{j=1}^{n}\left\{\left[\mu_{i} \min \left(k_{i}, m_{i}\right) u\left(k_{i}\right) p_{i j}+\theta_{i}\left(k_{i}-m_{i}\right) u\left(k_{i}-m_{i}\right) q_{i j}\right] v_{i}\left(k-I_{i}+I_{j}, t\right)+\right. \\
\left.+\left[\mu_{j} \min \left(k_{j}, m_{j}\right) u\left(k_{j}\right) p_{j i}+\theta_{j}\left(k_{j}-m_{j}\right) u\left(k_{j}-m_{j}\right) q_{j i}\right] v_{i}\left(k+I_{i}-I_{j}, t\right)\right\}+ \\
+\sum_{\substack{c, s=1 \\
c, s \neq i}}^{n}\left[\mu_{s} \min \left(k_{s}, m_{s}\right) u\left(k_{s}\right) p_{s c}+\theta_{s}\left(k_{s}-m_{s}\right) u\left(k_{s}-m_{s}\right) q_{s c}\right] v_{i}\left(k+I_{c}-I_{s}, t\right)+ \\
+\sum_{j=1}^{n}\left[\mu_{j} \min \left(k_{j}, m_{j}\right) u\left(k_{j}\right) p_{j i} r_{i j}\left(k+I_{i}-I_{j}, t\right)-\right. \\
-\mu_{i} \min \left(k_{i}, m_{i}\right) u\left(k_{i}\right) p_{i j} r_{j i}\left(k-I_{i}+I_{j}, t\right)+\theta_{j}\left(k_{j}-m_{j}\right) u\left(k_{j}-m_{j}\right) q_{j i} h_{i j}\left(k+I_{i}-I_{j}, t\right)+ \\
\left.+\theta_{i}\left(k_{i}-m_{i}\right) u\left(k_{i}-m_{i}\right) q_{i j} h_{j i}\left(k-I_{i}+I_{j}, t\right)\right]+\lambda p_{0 i} r_{0 i}\left(k+I_{i}, t\right)- \\
-\mu_{i} \min \left(k_{i}, m_{i}\right) u\left(k_{i}\right) p_{i 0} R_{i 0}\left(k-I_{i}, t\right)-\theta_{i}\left(k_{i}-m_{i}\right) u\left(k_{i}-m_{i}\right) q_{i 0} H_{i 0}\left(k-I_{i}, t\right)+r_{i}(k) .
\end{gathered}
$$

In this case

$$
\begin{aligned}
& A_{i}(k)=\sum_{j=1}^{n}\left[\mu_{j} \min \left(k_{j}, m_{j}\right) u\left(k_{j}\right) p_{j i} r_{i j}\left(k+I_{i}-I_{j}, t\right)-\mu_{i} \min \left(k_{i}, m_{i}\right) u\left(k_{i}\right) p_{i j} r_{j i}\left(k-I_{i}+I_{j}, t\right)+\right. \\
& \left.+\theta_{j}\left(k_{j}-m_{j}\right) u\left(k_{j}-m_{j}\right) q_{j i} h_{i j}\left(k+I_{i}-I_{j}, t\right)+\theta_{i}\left(k_{i}-m_{i}\right) u\left(k_{i}-m_{i}\right) q_{i j} h_{j i}\left(k-I_{i}+I_{j}, t\right)\right]+ \\
& +\lambda p_{0 i} r_{0 i}\left(k+I_{i}, t\right)-\mu_{i} \min \left(k_{i}, m_{i}\right) u\left(k_{i}\right) p_{i 0} R_{i 0}\left(k-I_{i}, t\right)- \\
& -\theta_{i}\left(k_{i}-m_{i}\right) u\left(k_{i}-m_{i}\right) q_{i 0} H_{i 0}\left(k-I_{i}, t\right)+r_{i}(k), \\
& \Lambda_{i}(k)=\lambda+\sum_{j=1}^{n}\left[\mu_{j} \min \left(k_{j}, m_{j}\right) u\left(k_{j}\right)\left(1-p_{j 0}\right)+\theta_{j}\left(k_{j}-m_{j}\right) u\left(k_{j}-m_{j}\right)\left(1-q_{j 0}\right)\right], \\
& \Phi_{i c s}(k)=\left\{\begin{array}{l}
\mu_{s} \min \left(k_{s}, m_{s}\right) u\left(k_{s}\right) p_{s c}+\theta_{s}\left(k_{s}-m_{s}\right) u\left(k_{s}-m_{s}\right) q_{s c}, \quad c, s=\overline{1, n}, \quad c, s \neq i, \\
{\left[\mu_{s} \min \left(k_{s}, m_{s}\right) u\left(k_{s}\right) p_{s i}+\theta_{s}\left(k_{s}-m_{s}\right) u\left(k_{s}-m_{s}\right) q_{s i}\right] \delta_{c i}, \quad c=\overline{1, n}, \quad c=i,} \\
{\left[\mu_{c} \min \left(k_{c}, m_{c}\right) u\left(k_{c}\right) p_{c i}+\theta_{c}\left(k_{c}-m_{c}\right) u\left(k_{c}-m_{c}\right) q_{c i}\right] \delta_{s i}, \quad c=\overline{1, n}, \quad s=i,} \\
\lambda p_{0 c}, \quad s=0, \\
\mu_{s} \min \left(k_{s}, m_{s}\right) u\left(k_{s}\right) p_{s 0}, \quad c=0 .
\end{array}\right.
\end{aligned}
$$


We use the successive approximations method combined with the series method to solve generalized system of DDE (1) and study properties of these approximations. The earlier successive approximations method was used for finding of expected incomes in systems of HM-network with multi-type massages of many classes [1, 2]. From (1) it follows

$$
\frac{d}{d t}\left\{e^{\Lambda_{i}(k) t} v_{i}(k, t)\right\}=e^{\Lambda_{i}(k) t}\left[\mathrm{~A}_{i}(k)+\sum_{c, s=1}^{n} \Phi_{i c s}(k) v_{i}\left(k+I_{c}-I_{s}, t\right)\right],
$$

thus

$$
v_{i}(k, t)=e^{-\Lambda_{i}(k) t}\left\{v_{i}(k, 0)+\int_{0}^{t} e^{\Lambda_{i}(k) x} \sum_{c, s=1}^{n} \Phi_{i c s}(k) v\left(k+I_{c}-I_{s}, x\right) d x\right\}+\frac{\mathrm{A}_{i}(k)}{\Lambda_{i}(k)}\left[1-e^{-\Lambda_{i}(k) t}\right]
$$

Let's suppose $v_{i m}(k, t)$ - approximation of income $v_{i}(k, t)$ at the $m$-th iteration, $v_{i m+1}(k, t)$ - solution of system (1), which was received by means of the successive approximations method, $m=0,1,2, \ldots$. Then from (2) it follows

$$
\begin{gathered}
v_{i m+1}(k, t)=e^{-\Lambda_{i}(k) t}\left\{v_{i}(k, 0)+\right. \\
\left.+\int_{0}^{t} e^{\Lambda_{i}(k) x} \sum_{c, s=1}^{n} \Phi_{i c s}(k) v_{i m}\left(k+I_{c}-I_{s}, x\right) d x\right\}+\frac{\mathrm{A}_{i}(k)}{\Lambda_{i}(k)}\left[1-e^{-\Lambda_{i}(k) t}\right] .
\end{gathered}
$$

It is obvious that $v_{i m}(k, 0)=v_{i}(k, 0)$, and let also $v_{i 0}(k, t)=v_{i}(k)=\lim _{t \rightarrow \infty} v_{i}(k, t)$. The stationary solution $v_{i}(k)$ of system (1) satisfies to relation

$$
v_{i}(k)=\frac{1}{\Lambda_{i}(k)}\left\{\mathrm{A}_{i}(k)+\sum_{c, s=1}^{n} \Phi_{i c s}(k) v_{i}\left(k+I_{c}-I_{s}\right)\right\}
$$

\section{Investigation of successive approximations of system incomes}

The following statements are true for successive approximations.

Theorem 2. Successive approximations $v_{i m}(k, t), m=1,2, \ldots$, for $t \rightarrow \infty$ converge to stationary solution of system (1), if it exists.

Proof. Expression for the first approximation is:

$$
v_{i 1}(k, t)=e^{-\Lambda_{i}(k) t} v_{i}(k, 0)+\frac{1}{\Lambda_{i}(k)}\left[\mathrm{A}_{i}(k)+\sum_{c, s=1}^{n} \Phi_{i c s}(k) v_{i 0}\left(k+I_{c}-I_{s}, t\right)\right]\left[1-e^{-\Lambda_{i}(k) t}\right] .
$$


Using (4), we obtain $\lim _{t \rightarrow \infty} v_{i 1}(k, t)=v_{i}(k)$. Let's assume $\lim _{t \rightarrow \infty} v_{i m}(k, t)=v_{i}(k)$. Then using (3), (4) and L'Hospital's rule the next expression occurs:

$$
\begin{gathered}
\lim _{t \rightarrow \infty} v_{i m+1}(k, t)=\frac{\mathrm{A}_{i}(k)}{\Lambda_{i}(k)}+\lim _{t \rightarrow \infty} \frac{\int_{0}^{t} e^{\Lambda(k) x} \sum_{c, s=1}^{n} \Phi_{i c s}(k) v_{i m}\left(k+I_{c}-I_{s}, x\right) d x}{e^{\Lambda_{i}(k) t}}= \\
=\frac{\mathrm{A}_{i}(k)}{\Lambda_{i}(k)}+\frac{1}{\Lambda_{i}(k)} \lim _{t \rightarrow \infty} \sum_{c, s=1}^{n} \Phi_{i c s}(k) v_{i m}\left(k+I_{c}-I_{s}, t\right)=v_{i}(k) .
\end{gathered}
$$

Thus using the method of mathematical induction, we obtain the statement of the theorem.

Theorem 3. Sequence $\left\{v_{i m}(k, t)\right\}, m=0,1,2, \ldots$, which was built by scheme (3), converges to unique solution of system (1) for $m \rightarrow \infty$ and any initial approximation $v_{i 0}(k, t)$ bounded by $t$.

Proof. Since $v_{i 0}(k, t)$ is a bounded function, so $v_{i 1}(k, t)$ is also bounded function according to (3), thus we may write

$$
\left|v_{i 1}(k, t)-v_{i 0}(k, t)\right| \leq C_{i}(k),
$$

where $C_{i}(k)$ - some constant which doesn't depend on $t$. Let's prove

$$
\left|v_{i m}(k, t)-v_{i m-1}(k, t)\right| \leq C_{i}(k) \varphi_{i}^{m-1}(k) \frac{t^{m-1}}{(m-1) !},
$$

where $\varphi_{i}(k)=\sum_{c, s=1}^{n} \Phi_{i c s}(k)$. According to (5) inequality (6) is true for $m=1$. Let's suppose that inequality (6) is true for some $m=N>1$ and let's prove that (6) is right for $m=N+1$, using (3). Taking into consideration (2), (6) we obtain:

$$
\begin{gathered}
\left|v_{i N+1}(k, t)-v_{i N}(k, t)\right|= \\
=e^{-\Lambda_{i}(k) t} \int_{0}^{t} e^{\Lambda_{i}(k) x} \sum_{c, s=1}^{n} \Phi_{i c s}(k)\left|v_{i N}\left(k+I_{c}-I_{s}, x\right)-v_{i N-1}\left(k+I_{c}-I_{s}, x\right)\right| d x \leq \\
\leq e^{-\Lambda_{i}(k) t} \int_{0}^{t} e^{\Lambda_{i}(k) x} \varphi_{i}(k) C_{i}(k) \varphi_{i}^{N-1}(k) \frac{x^{N-1}}{(N-1) !} d x \leq C_{i}(k) \varphi_{i}^{N}(k) \int_{0}^{t} \frac{x^{N-1}}{(N-1) !} d x=C_{i}(k) \varphi_{i}^{N}(k) \frac{t^{N}}{N !},
\end{gathered}
$$

i.e. inequality (6) is true. 
Since

$$
\begin{gathered}
\lim _{m \rightarrow \infty} v_{i m}(k, t)=\lim _{m \rightarrow \infty}\left\{v_{i 0}(k, t)+\sum_{N=1}^{m}\left[v_{i N}(k, t)-v_{i N-1}(k, t)\right]\right\}= \\
=v_{i 0}(k, t)+\sum_{N=1}^{\infty}\left[v_{i N}(k, t)-v_{i N-1}(k, t)\right] \leq \\
\leq v_{i 0}(k, t)+C_{i}(k) \sum_{N=1}^{\infty} \frac{\left[\varphi_{i}(k) t\right]^{N-1}}{(N-1) !} \leq v_{i 0}(k, t)+C_{i}(k) e^{\varphi_{i}(k) t},
\end{gathered}
$$

so limit of sequence $\left\{v_{i m}(k, t)\right\}, m=0,1,2, \ldots$, exists and we define it as $v_{i \infty}(k, t)$.

If we put $v_{i \infty}(k, t)$ in (3) instead of $v_{i m+1}(k, t)$ and $v_{i m}(k, t)$, it will be seen that $v_{i \infty}(k, t)$ is the solution of the system and it satisfies the initial conditions $v_{i \infty}(k, 0)=v_{i}(k, 0)$ according to the previous theorem 2 .

Let's prove that found solution is unique. Let's suppose that different solution $v_{i}^{*}(k, t)$ exists. If we replace $v_{i}(k, t), v_{i}(k, 0)$ and $v_{i}\left(k+I_{c}-I_{s}, x\right)$ in (2) by $v_{i}^{*}(k, t)$, $v_{i}^{*}(k, 0)$ and $v_{i}^{*}\left(k+I_{c}-I_{s}, x\right)$ correspondently, then relation (2) is satisfied for $v_{i}^{*}(k, t)$. Thus using (3) we obtain

$$
\begin{gathered}
\left|v_{i m}(k, t)-v_{i}^{*}(k, t)\right| \leq e^{-\Lambda_{i}(k) t}\left|v_{i}(k, 0)-v_{i}^{*}(k, 0)\right|+ \\
+\int_{0}^{t} e^{-\Lambda_{i}(k)(t-x)} \sum_{c, s=1}^{n} \Phi_{i c s}(k)\left|v_{i m}\left(k+I_{c}-I_{s}, x\right)-v_{i}^{*}\left(k+I_{c}-I_{s}, x\right)\right| d x .
\end{gathered}
$$

Similar to the proof of inequality (5) we can prove that

$$
\left|v_{i m}(k, t)-v_{i}^{*}(k, t)\right| \leq M_{i}(k) \varphi_{i}^{m}(k) \frac{t^{m}}{m !},
$$

where $M_{i}(k)$ doesn't depend on $t$. The right part of this inequality tends towards zero as common term of converge series $\sum_{m=0}^{\infty} M_{i}(k) \varphi_{i}^{m}(k) \frac{t^{m}}{m !}=M_{i}(k) e^{\varphi_{i} t}$, thus $\lim _{m \rightarrow \infty} v_{i m}(k, t)=v_{i}^{*}(k, t)$. Earlier we have proved that $\lim _{m \rightarrow \infty} v_{i m}(k, t)=v_{i}(k, t)$, so $v_{i}(k, t)=v_{i}^{*}(k, t)$ and it proves the unique of solution.

The next statement is more useful for practice. 
Theorem 4. Any approximation $v_{i m}(k, t), m \geq 1$, can be presented in form of power series

$$
v_{i m}(k, t)=\sum_{l=0}^{\infty} d_{i m l}(k) t^{l},
$$

whose coefficients satisfy the recurrence relations:

$$
\begin{gathered}
d_{i m+1 l}(k)=\frac{\left[-\Lambda_{i}(k)\right]^{l}}{l !}\left\{v_{i}(k, 0)-\frac{\mathrm{A}_{i}(k)}{\Lambda_{i}(k)}+\sum_{u=0}^{l-1} \frac{(-1)^{u+1} D_{i m u}(k)}{\left[\Lambda_{i}(k)\right]^{u+1}} u !\right\}, l \geq 0, \\
d_{i m+10}(k)=v_{i}(k, 0), \quad d_{i 0 l}(k)=v_{i}(k, 0) \delta_{l 0},
\end{gathered}
$$

where

$$
D_{i m l}(k)=\sum_{c, s=1}^{n} \Phi_{i c s}(k) d_{i m l}\left(k+I_{c}-I_{s}\right),
$$

$\delta_{l 0}-$ Kronecker's symbol. If $\Lambda_{i}(k) \geq 1$, series (7) converges for any finite $t>0$.

Proof. Let's prove that the coefficients of series (7) satisfy the recurrence relations (8). If we put successive approximations (7) in (3) and consider

$$
e^{-\Lambda_{i}(k) t} \int_{0}^{t} e^{\Lambda_{i}(k) x} x^{l} d x=\left[\frac{1}{\Lambda_{i}(k)}\right]^{l+1} l ! \sum_{j=l+1}^{\infty} \frac{\left[-\Lambda_{i}(k) t\right]^{j}}{j !}, l=0,1,2, \ldots,
$$

we will obtain

$$
\begin{gathered}
\sum_{l=0}^{\infty} d_{i m+1 l}(k) t^{l}=\frac{\mathrm{A}_{i}(k)}{\Lambda_{i}(k)}\left[1-e^{-\Lambda_{i}(k) t}\right]+e^{-\Lambda_{i}(k) t} v_{i}(k, 0)+ \\
+\sum_{l=0}^{\infty} \sum_{c, s=1}^{n} \Phi_{i c s}(k) d_{i m l}\left(k+I_{c}-I_{s}\right)\left[\frac{-1}{\Lambda_{i}(k)}\right]^{l+1} l ! \sum_{u=l+1}^{\infty} \frac{\left[-\Lambda_{i}(k) t\right]^{u}}{u !} .
\end{gathered}
$$

Taking into account definition (9) this series can be rewritten in form:

$$
\begin{gathered}
\sum_{l=0}^{\infty} d_{i m+1 l}(k) t^{l}=\frac{\mathrm{A}_{i}(k)}{\Lambda_{i}(k)}+e^{-\Lambda_{i}(k) t}\left[v_{i}(k, 0)-\frac{\mathrm{A}_{i}(k)}{\Lambda_{i}(k)}\right]+ \\
+\sum_{l=0}^{\infty} D_{i m l}(k)\left[\frac{-1}{\Lambda_{i}(k)}\right]^{l+1} l ! \sum_{u=l+1}^{\infty} \frac{(-1)^{u}\left[\Lambda_{i}(k)\right]^{u}}{u !} t^{u} .
\end{gathered}
$$


Interchanging summation indices and making series expansion of function $e^{-\Lambda_{i}(k) t}$ in powers of $t$, we obtain

$$
\sum_{l=0}^{\infty} d_{i m+1 l}(k) t^{l}=\frac{\mathrm{A}_{i}(k)}{\Lambda_{i}(k)}+\sum_{l=0}^{\infty} \frac{\left[-\Lambda_{i}(k)\right]^{l}}{l !}\left\{v_{i}(k, 0)-\frac{\mathrm{A}_{i}(k)}{\Lambda_{i}(k)}+\sum_{u=0}^{l-1} \frac{(-1)^{u+1} D_{i m u}(k)}{\left[\Lambda_{i}(k)\right]^{u+1}} u !\right\} t^{l} .(10)
$$

If we equate coefficients by $t^{l}$ in the left and the right parts of expression (10), we will obtain recurrence relations (8) for coefficients of power series (7).

To find the converge radius $R_{i}(k)$ of power series (7) we use the Cauchy-Hadamard formula:

$$
\frac{1}{R_{i}(k)}=\lim _{l \rightarrow \infty} \sqrt[l]{\left|d_{i m l}(k)\right|}
$$

From (8) it follows that

$$
\begin{aligned}
\left|d_{i m l}(k)\right| & =\frac{\left[\Lambda_{i}(k)\right]^{l}}{l !}\left|v_{i}(k, 0)-\frac{\mathrm{A}_{i}(k)}{\Lambda_{i}(k)}+\sum_{u=0}^{l-1} \frac{(-1)^{u+1} D_{i m-1 u}(k)}{\left[\Lambda_{i}(k)\right]^{u+1}} u !\right| \leq \\
& \left.\leq \frac{\left[\Lambda_{i}(k)\right]^{l}}{l !}|| v_{i}(k, 0)|+| \frac{\mathrm{A}_{i}(k)}{\Lambda_{i}(k)}\left|+\sum_{u=0}^{l-1}\right| \frac{D_{i m u}(k)}{\left[\Lambda_{i}(k)\right]^{u+1}} u ! \mid\right) .
\end{aligned}
$$

Let's prove that $\left|D_{i m u}(k)\right|, m \geq 1, u=\overline{0, l-1}$, is bounded by some finite value $C_{i}(k)$. If $v_{i}(k, 0)$ is bounded then relation $D_{i 00}(k)=\sum_{c, s=1}^{n} \Phi_{i c s}(k) v_{i 0}\left(k+I_{c}-I_{s}, 0\right)$ $\leq C_{i 00}(k)$ follows from definition of $D_{i m u}(k), C_{i 00}(k)$ - some bounded value and $D_{i 0 l}(k)=0$ for each $l=1,2, \ldots$. Since $D_{i m-10}(k)=D_{i m-20}(k)=\ldots=D_{i 10}(k)=D_{i 00}(k)$, so $D_{i m-10}(k) \leq C_{i 00}(k), m \geq 1$.

Using induction we can prove that

$$
\left|D_{i m-1 l}(k)\right| \leq \frac{C_{i m-1 l}(k)}{l !}, l=1,2, \ldots
$$

For $l=1$ :

$$
\left|D_{i m-11}(k)\right| \leq \sum_{c, s=1}^{n} \Phi_{i c s}(k) d_{i m-11}\left(k+I_{c}-I_{s}\right)=
$$




$$
\begin{gathered}
=\frac{1}{1 !} \sum_{c, s=1}^{n} \Phi_{i c s}(k) \Lambda\left(k+I_{c}-I_{s}\right)\left|v_{i}\left(k+I_{c}-I_{s}, 0\right)-\frac{\mathrm{A}_{i}\left(k+I_{c}-I_{s}\right)}{\Lambda_{i}\left(k+I_{c}-I_{s}\right)}-\frac{D_{i m-20}\left(k+I_{c}-I_{s}\right)}{\Lambda_{i}\left(k+I_{c}-I_{s}\right)}\right|= \\
=\frac{1}{1 !} \sum_{c, s=1}^{n} \Phi_{i c s}(k)\left|v_{i}\left(k+I_{c}-I_{s}, 0\right) \Lambda_{i}\left(k+I_{c}-I_{s}\right)-\mathrm{A}_{i}\left(k+I_{c}-I_{s}\right)-D_{i m-20}\left(k+I_{c}-I_{s}\right)\right| \leq \\
\leq \frac{C_{i m-11}(k)}{1 !},
\end{gathered}
$$

where $C_{i m-11}(k)$ is bounded value. Let's assume that inequality (12) is true for $l-1$, i.e.

$$
\left|D_{i m-1 l-1}(k)\right| \leq \frac{C_{i m-1 l-1}(k)}{(l-1) !} .
$$

Let's prove that (12) is satisfied for $l$. Using (9) we obtain

$$
\begin{gathered}
\left|D_{i m-1 l}(k)\right| \leq \sum_{c, s=1}^{n} \Phi_{i c s}(k)\left|d_{i m-1 l}\left(k+I_{c}-I_{s}\right)\right| \leq \frac{1}{l !} \sum_{c, s=1}^{n} \Phi_{i c s}(k)\left[\Lambda_{i}\left(k+I_{c}-I_{s}\right)\right]^{l} \times \\
\left|v_{i}\left(k+I_{c}-I_{s}, 0\right)-\frac{\mathrm{A}_{i}\left(k+I_{c}-I_{s}\right)}{\Lambda_{i}\left(k+I_{c}-I_{s}\right)}+\sum_{u=0}^{l-1} \frac{(-1)^{u+1} D_{i m-2 u}\left(k+I_{c}-I_{s}\right) u !}{\left[\Lambda_{i}\left(k+I_{c}-I_{s}\right)\right]^{u+1}}\right| .
\end{gathered}
$$

Thus according to (13) we can take the right part of the last inequality as $C_{m-1 l}(k)$.

Let $C_{i 1}(k)=\max _{m, l} C_{i m-1 l}(k)$, then

$$
\begin{gathered}
\sum_{u=0}^{l-1}\left|\frac{D_{i m u}(k)}{\left[\Lambda_{i}(k)\right]^{u+1-l} l !} u !\right| \leq \sum_{u=0}^{l-1} \frac{C_{i m u}(k) u !}{\left[\Lambda_{i}(k)\right]^{u+1-l} l ! u !} \leq \\
\leq \frac{C_{i 1}(k)\left[\Lambda_{i}(k)\right]^{l}}{l !} \sum_{u=0}^{l-1} \frac{1}{\left[\Lambda_{i}(k)\right]^{u+1}}=\frac{C_{i 1}(k)\left[\Lambda_{i}(k)\right]^{l}}{l !} S_{i l-1}(k),
\end{gathered}
$$

where $S_{i l-1}(k)$ - sum of the first of $l$ terms of geometric progression, $S_{i l-1}(k)=\left\{\begin{array}{cc}\frac{\left[\Lambda_{i}(k)\right]^{l}-1}{\left[\Lambda_{i}(k)\right]^{l}\left(\Lambda_{i}(k)-1\right)}, & \Lambda_{i}(k) \neq 1, \\ l, & \Lambda_{i}(k)=1 .\end{array}\right.$ 


$$
\begin{aligned}
& \frac{1}{R_{i}(k)} \leq \Lambda_{i}(k) \lim _{l \rightarrow \infty} l \sqrt[l]{\frac{1}{l !}\left|v_{i}(k, 0)\right|+\left|\frac{\mathrm{A}_{i}(k)}{\Lambda_{i}(k)}\right|+\sum_{u=0}^{l-1}\left|\frac{D_{i m u}(k)}{\left[\Lambda_{i}(k)\right]^{u+1}} u !\right|} \leq \\
& \leq \Lambda_{i}(k) \lim _{l \rightarrow \infty} \sqrt[l]{\frac{1}{(l-1) !}} \lim _{l \rightarrow \infty} \sqrt[l]{\left|\frac{v_{i}(k, 0)}{l}\right|+\left|\frac{\mathrm{A}_{i}(k)}{\Lambda_{i}(k) l}\right|+\frac{C_{i}(k)}{l} S_{i l-1}(k) .}
\end{aligned}
$$

It is obvious that the first and the second summands under the square root in the second limit tends toward zero for $l \rightarrow \infty$. The last summand

$$
\begin{aligned}
\frac{C_{i}(k) S_{i l-1}(k)}{l} & =\left\{\begin{array}{cc}
C_{i}(k) \frac{\left[\Lambda_{i}(k)\right]^{l-2}-1}{l\left[\Lambda_{i}(k)\right]^{l-2}\left(\Lambda_{i}(k)-1\right)}, & \Lambda_{i}(k) \neq 1, \\
C_{i}(k), & \Lambda_{i}(k)=1,
\end{array}\right. \\
& =\left\{\begin{array}{cc}
1-\frac{1}{\left[\Lambda_{i}(k)\right]^{l-2}}, & \Lambda_{i}(k) \neq 1, \\
C_{i 1}(k) \frac{1\left(\Lambda_{i}(k)-1\right)}{C_{i}(k),} & \Lambda_{i}(k)=1,
\end{array}\right.
\end{aligned}
$$

is bounded if $\Lambda_{i}(k) \geq 1$.

Let's show that equation (15) is satisfied for $\Lambda_{i}(k) \geq 1$

$$
\lim _{l \rightarrow \infty} \sqrt[l]{\left|\frac{v_{i}(k, 0)}{l}\right|+\left|\frac{\mathrm{A}_{i}(k)}{\Lambda_{i}(k) l}\right|+\frac{C_{i}(k)}{l} S_{i l-1}(k)}=1 .
$$

Let's consider the cases:

1) Let $\quad a_{i l}(k)=\left|\frac{v_{i}(k, 0)}{l}\right|+\left|\frac{\mathrm{A}_{i}(k)}{\Lambda_{i}(k) l}\right|+\frac{C_{i}(k)}{l} S_{i l-1}(k) \quad$ and $\quad a_{i l}(k) \geq 1, \quad$ suppose $\sqrt[l]{a_{i l}(k)}=1+x_{i l}(k), \quad x_{i l}(k) \geq 0$. According to Bernoulli's inequality $a_{i l}(k)=$ $=\left(1+x_{i l}(k)\right)^{l} \geq 1+l x_{i l}(k)$, so

$$
0 \leq x_{i l}(k) \leq \frac{a_{i l}(k)-1}{l}, \quad 0 \leq \lim _{l \rightarrow \infty} x_{i l}(k) \leq \lim _{l \rightarrow \infty} \frac{a_{i l}(k)-1}{l}=0,
$$

i.e. $\lim _{l \rightarrow \infty} x_{i l}(k)=0$ and $\lim _{l \rightarrow \infty} \sqrt[l]{a_{i l}(k)}=1+0=1$. 
2) Let $0<a_{i l}(k)<1$, then $\frac{1}{a_{i l}(k)}>1$ and

$$
\lim _{l \rightarrow \infty} \sqrt[l]{a_{i l}(k)}=\lim _{l \rightarrow \infty} \frac{1}{\sqrt[l]{1 / a_{i l}(k)}}=\frac{1}{\lim _{l \rightarrow \infty} \sqrt[l]{1 / a_{i l}(k)}}=1 .
$$

Thus relation (15) is satisfied.

Let's consider $\lim _{l \rightarrow \infty} \sqrt[l]{\frac{1}{(l-1) !}}$. Using Stirling's formula we obtain:

$$
\begin{aligned}
& \lim _{l \rightarrow \infty} \sqrt[l]{\frac{1}{(l-1) !}}=\frac{1}{\lim _{l \rightarrow \infty} \sqrt[l]{(l-1) !}}=\frac{1}{\lim _{l \rightarrow \infty} \sqrt[l]{\sqrt{2 \pi(l-1)}\left(\frac{l-1}{e}\right)^{l-1} \exp \left(\frac{\theta}{12(l-1)}\right)}}= \\
& =\frac{1}{\exp \left\{\lim _{l \rightarrow \infty} \ln \left[(2 \pi(l-1))^{\frac{1}{2 l}}(l-1)^{\frac{l-1}{l}} \exp \left(\frac{\theta}{12(l-1) l}-\frac{l-1}{l}\right)\right]\right\}}= \\
& =\frac{1}{\exp \left\{\lim _{l \rightarrow \infty}\left[\frac{1}{2 l} \ln (2 \pi(l-1))+\frac{l-1}{l} \ln (l-1)+\left(\frac{\theta}{12(l-1) l}-\frac{l-1}{l}\right)\right]\right\}},
\end{aligned}
$$

where $0<\theta<1$. Obviously that $\lim _{l \rightarrow \infty} \frac{1}{2 l} \ln (2 \pi(l-1))=0, \lim _{l \rightarrow \infty} \frac{l-1}{l} \ln (l-1)=\infty$, so $\lim _{l \rightarrow \infty} \sqrt[l]{\frac{1}{(l-1) !}}=\frac{1}{\infty}=0$.

From (14) it follows that the converge radius of power series (7) equals $\infty$.

Thus we proved that every approximation $v_{i m}(k, t)$ can be presented in form of the power series which converges for $\Lambda_{i}(k) \geq 1$ and found recurrence relations (8), (9) for coefficients of this series which are useful for computer calculations.

The last theorem allows us to find expected incomes of systems of the queueing network with a huge number of states within a reasonable time. Besides, it is necessary to note that the present method can be applied to find expected incomes of systems of the open queueing network with an infinite number of states.

\section{Example}

Let's consider the closed HM-network with one-type messages, number of messages in the network is $K=8$. Let $n=5, m_{i}=1, i=\overline{1,4}, m_{5}=2$. The state number 
of such network equals 495. Let also $\mu_{1}=\mu_{2}=\mu_{3}=\mu_{4}=2, \mu_{5}=4, p_{j 5}=1$, $p_{5 j}=\frac{1}{4}, \quad j=\overline{1,4}, \quad$ the $\quad$ rest of probabilities are equal zero; $\theta_{1}=0.2, \theta_{2}=\theta_{3}=\theta_{4}=0.5, \theta_{5}=1, q_{j 5}=1, q_{5 j}=\frac{1}{4}, j=\overline{1,4}$, the rest of probabilities are equal zero; $r_{i}(k)=k_{i}+1, r_{i j}(k, t)=k_{i} t, h_{i j}(k, t)=k_{i} \sqrt{t}, i=\overline{1,5}$. The income change of the third system by time is presented on plot 1 . Income was calculated by means of computer with the help of relations (7)-(9), the initial state of system was $(0,1,2,0,5)$.

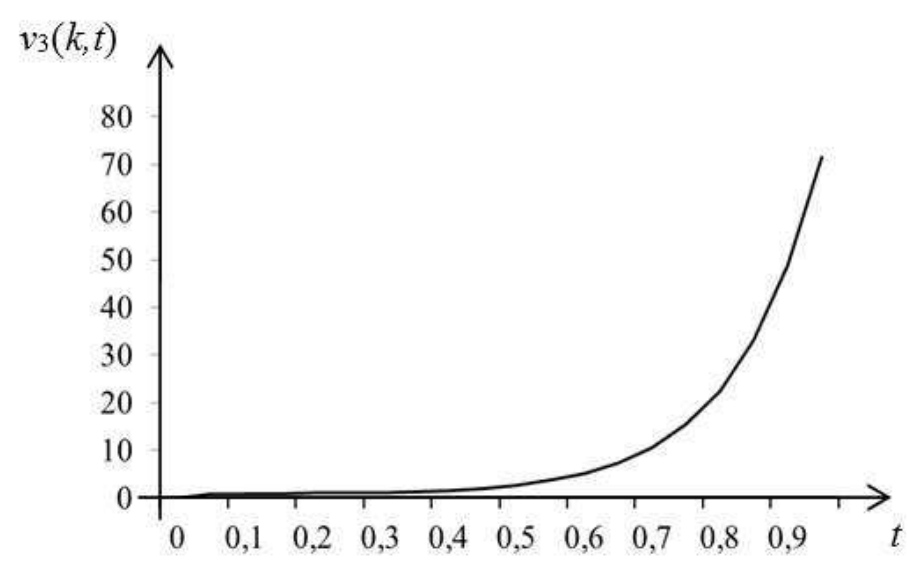

Fig. 1. Income change of system $S_{3}$ in interval $[0,1]$

\section{References}

[1] Matalytski M., On some result in analysis and optimization of Markov network with incomes and their applications, J. Automation and Remote Control. 2009, 10(70), 1683-1697.

[2] Matalytski M., Koluzaeva E., Investigation of Markov HM-networks with multi-type messages of many classes by means of successive approximations method, Proc. of the National Academy of Sciences of Belarus, Phys.-Math. Sciences 2008, 4, 113-119. 